\title{
Diagnóstico del sistema de instrucción durante pandemia Covid-19. Área físico-matemática de Escuela de Física de la UNAH
}

\author{
Diagnosis of the training system during the Covid-19 pandemic. \\ Physical-mathematical area of the School of Physics at UNAH
}

F. BARRALAGA ${ }^{1}$

Recibido: 15 de octubre de 2020 / Aceptado: 18 de diciembre de 2020

\begin{abstract}
RESUMEN
Todas las sociedades a nivel global están afrontando una crisis en todos los ámbitos de su actividad, causada por la pandemia Covid-19. Muchas de las implicaciones resultantes de la dinámica de afrontamiento serán definidas y analizadas aún después de que la crisis haya sido superada, y con seguridad encontraremos que la capacidad de adaptación de la especie humana, habrá sido la impulsora de este proceso de superación. Se realizó un diagnóstico del sistema de instrucción virtual, que surgió como respuesta de adaptación ante esta crisis en la Escuela de Física de la Universidad Nacional Autónoma de Honduras, UNAH. Se consideran en este artículo los hallazgos más relevantes observados en esta evaluación, particularmente en el sector estudiantil del área físico-matemática, es decir, los estudiantes de ingenierías y ciencias.
\end{abstract}

\begin{abstract}
Every society at global level, is affronting a crisis at all over their whole activities, caused for the pandemic process generated by Covid-19. Some of the implications resulting from the dynamic of affronting this pandemic issue, will be defined and analyzed, even after the crisis had been overcome, and surely we will find, that the adaptation capacity of human kind, was the inner impulse needed to get the solution.

A diagnosis of the virtual instruction system, risen as the adaptation response against the crisis, at the Physics School of the Autonomous University of Honduras, UNAH, was realized. In this paper are considered the most relevant findings observed in this evaluation, that was particularly applied to the student sector of the Physic-Mathematic area, that is to say, engineering and sciences students.
\end{abstract}

\section{PALABRAS CLAVES}

Instrucción virtual, adaptabilidad ante Covid-19, resiliencia educativa

KEYWORDS

Virtual instruction, adaptability against Covid-19, educative resilience
${ }^{1}$ Escuela de Física, Facultad de Ciencias,

Universidad Nacional Autónoma de Honduras email: francisco.barralaga@unah.edu.hn 


\section{I | INTRODUCCIÓN}

La educación actualmente está intimamente relacionada con los sistemas multimedia(Gonzáles, 2020). Esto es cierto en todos los niveles de instrucción, y probablemente su máxima aplicabilidad se observe a nivel superior.

La crisis educativa provocada por la pandemia de Covid-19, según reporta la UNESCO(UNESCO, 2020) no ha impedido que muchos países latinoamericanos hayan continuado con la programación original planificada. En Honduras a partir del 16 de marzo del 2020 la sociedad inició un inmediato y casi absoluto distanciamiento; el mismo fue exitosamente afrontado por la Escuela de Física de la UNAH gracias a la familiaridad que tenían los docentes y estudiantes con los sistemas multimedia. Esto sin embargo requirió para otras escuelas, la implementación de un proceso emergente de capacitación y desarrollo de habilidades por parte tanto de docentes como de sus estudiantes.

Varias unidades de la UNAH realizaron un seguimiento de la etapa crítica inicial de este proceso de afrontamiento; particularmente la Escuela de Física, llevó a cabo un diagnóstico tanto del sector estudiantil del área físico-matemátice ${ }^{1}$ como del área correspondiente a la salud ${ }^{2}$, con el objetivo de determinar aspectos relevantes que pudieran ser útiles para las autoridades a cargo de la toma de decisiones en temas de capacitación a docentes y estudiantes, selección de herramientas u objetos de instrucción, o aspectos tecnológicos y económicos institucionales.

Un sistema de instrucción es complejo, abarca el ámbito de los docentes, el de los estudiantes y el de la institución misma. En esta publicación se incluyen únicamente los aspectos del sector estudiantil, resultantes de una consulta realizada a un grupo de estudiantes del área de servicio físico-matemática, es decir, estudiantes de ingenierías, física, matemáticas y astronomía. La consulta fue contestada por 171 estudiantes de las asignaturas Física General I (FS-100) y Física General II (FS-200). Se decidió que la consulta fuera anónima, para permitir que las opiniones de los jóvenes fuesen totalmente libres y espontáneas.

Se incluyen en este informe los aspectos más relevantes del análisis de los resultados con la esperanza de que los mismos permitan identificar factores importantes en futuras decisiones relativas al afrontamiento institucional de esta pandemia.

\section{I DESARROLLO DEL TEMA}

La investigación realizada fue del tipo cuali-cuantitativa, porque el instrumento de consulta o medición utilizado incluyó tanto respuestas de cacterización numérica, además de preguntas abiertas que requirieron la aplicación de herramientas de análisis cualitativo.

La consulta fue aplicada a una población estudiantil de 850 estudiantes matriculados en las asignaturas de Física General I y Física General II (FS-100 y FS-200 respectivamente), en la etapa final del primer período académico del 2020, momento para el que los consultados habían tenido suficiente experiencia con el sistema de instrucción emergente implementado en la Escuela de Física de la UNAH, estaban

\footnotetext{
${ }^{1}$ Estudiantes de ingenierías y Ciencias

${ }^{2}$ Estudiantes de ciencias médicas y biológicas
} 
familiarizados con el comportamiento de la plataforma tecnológica sobre la que se implementó esta instrucción, y eran plenamente conscientes del aislamiento social impuesto por las autoridades como medida de acción contra la pandemia.

Para el cálculo de la muestra se aplicó la fórmula de población finita(y Roldán S. F., 2015). Los detalles son los siguientes:

$$
\mathrm{n}=\frac{\sigma^{2} z^{2} N}{e^{2}(N-1)+\sigma^{2} z}
$$

con

$$
\begin{aligned}
e & =0.09, \\
\sigma & =0.5, \\
N & =850, \\
z & =1.96
\end{aligned}
$$

Resultando en $n=105$ estudiantes.

Contestaron 171 estudiantes, matriculados en dos de las cuatro asignaturas consultadas, sin embargo, FS-321 (Electricidad y Magnetismo I) y FS-415 (Electricidad y Magnetismo II), cuyos alumnos no contestaron, es un grupo de alumnos que corresponde a menos del $15 \%$ de la población del área físico-matemática atendida, por lo que los resultados y estimaciones obtenidas pueden ser considerados válidos para toda la población.

Las respuestas fueron totalmente libres y espontáneas. La consulta contenía 23 preguntas separadas en 5 secciones, que consideraban los aspectos relevantes del ámbito estudiantil en este proceso de instrucción, es decir:

- Equipo de cómputo y conexión a internet,

- Competencias requeridas,

- Técnología de comunicación,

- Desempeño de plataforma tecnológica,

- Posibles herramientas de instrucción.

Google Docs, la herramienta de consulta utilizada para la creación y distribución de la consulta, ofrece un resumen de los resultados que incluye variedad de despliegues gráficos de las tendencias de las respuestas y un archivo opcional para el análisis independiente.

Aunque algunos gráficos del formulario de Google Docs han sido incluidos en el análisis de los resultados, se optó por hacer uso del archivo .cs ${ }^{3}$ para realizar un análisis de resultados independiente 


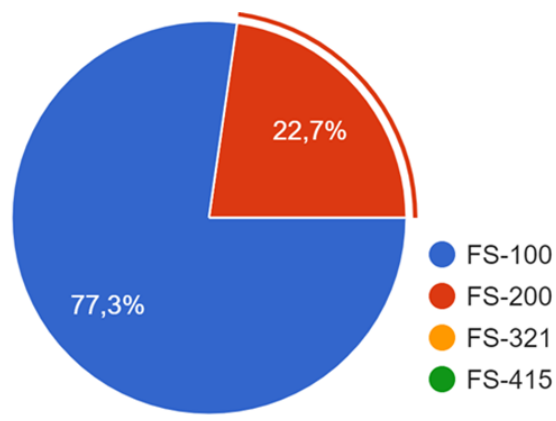

Figura 1: Distribución porcentual de asignaturas de muestra.

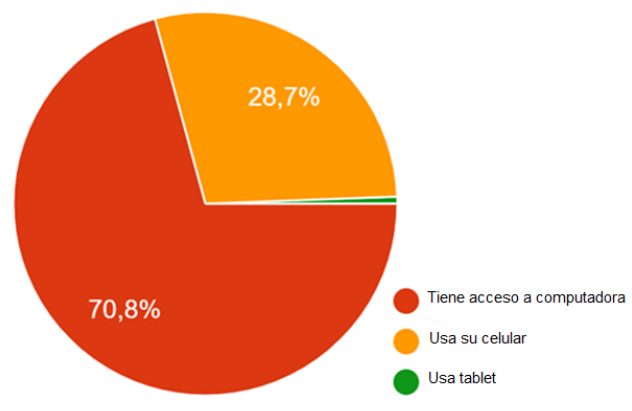

Figura 2: Disposición de equipo de computo.

de Google Docs, aplicando herramientas de Machine Learning con librerías muy conocidas en el lenguaje Python 3 (Acevedo, 2020, Gallery, 2020). En el siguiente apartado, se detallan los aspectos más relevantes del resultado del análisis de esta consulta, estructurándose así, un diagnóstico objetivo del sistema de instrucción implementado por la Escuela de Física.

\section{III | RESULTADOS OBTENIDOS}

\section{I Área académica de la población}

La Figura 1 muestra los porcentajes de las dos asignaturas que cuyos estudiantes respondieron.

\section{2 | Equipo y Conexión de Internet}

En la figura 2, se aprecia la distribución porcentual del acceso a equipo de computo por parte de los estudiantes consultados.

La gráfica de la Figura 3 indica los porcentajes de acceso a servicios de internet. Casi un $65 \%$ contaban con acceso a internet habiendo una carencia de más del $30 \%$ de este servicio. Por otra parte la Figura 4 señala el rango de inversión que las familias de estos estudiantes sin acceso a conexión de internet tienen que hacer, al verse obligados a usar su celular.

\section{I Competencias requeridas}

En cuanto a las competencias requeridas por los consultados, necesarias para afrontar una instrucción exclusivamente virtual, las respuestas indicadas en la Figura 5 muestran que más del $65 \%$ afirma no tener dificultades para llevar a cabo este tipo de instrucción. Adicionalmente la gráfica de la Figura 6 muestra que menos del $16 \%$ manifiesta tener dificultades para el manejo de las aplicaciones de computo involucradas en la instrucción. 


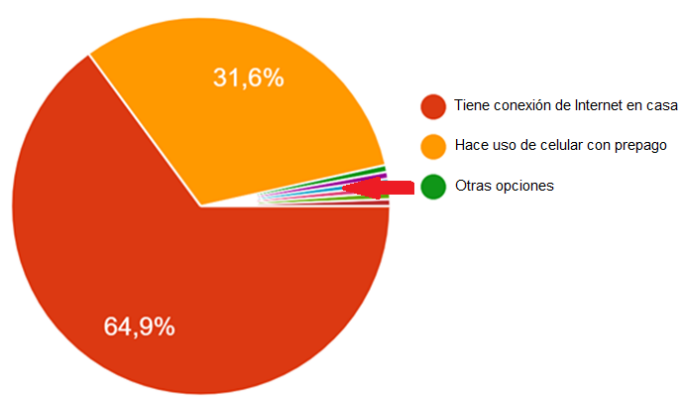

Figura 3: Acceso a Internet.

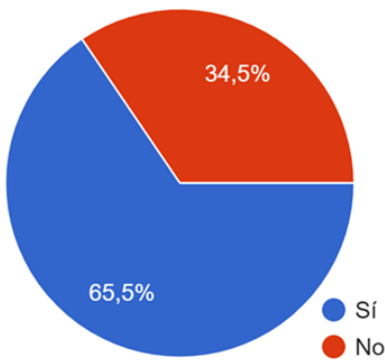

Figura 5: Disposición de competencias para la instrucción virtual.

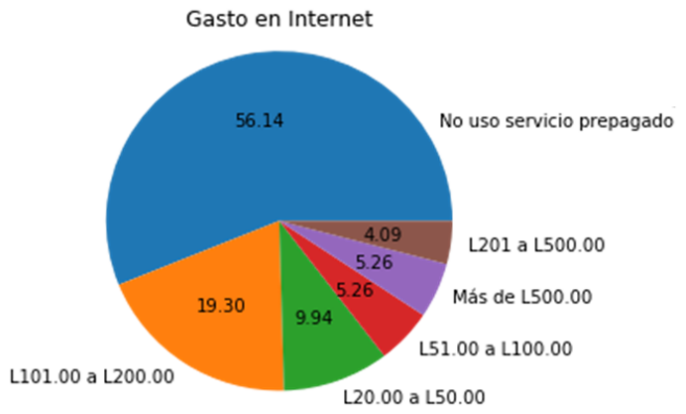

Figura 4: Gasto familiar del sector sin acceso a Internet.

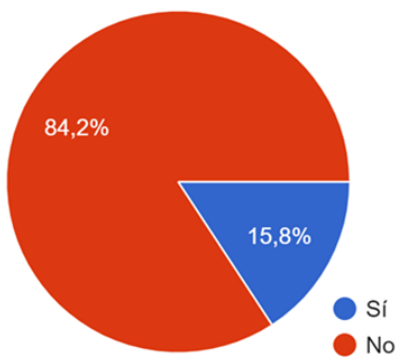

Figura 6: Manejo de herramientas de cómputo.

\section{4| Tecnológia de Comunicación}

El $63.2 \%$ de los docentes utilizan alguna aplicación de comunicación sincrónica para establecer contacto con sus estudiantes en sus labores de instrucción, estos resultados se incluyen en Figura 7 Zoom es la herramienta de comunicación sincrónica que mayormente utilizan los docentes, un $85.2 \%$ de los docentes la utilizan como se indica en la Figura 8 .

Adicionalmente, los docentes hacen uso de otras herramientas de conexión asíncrona para complementar la comunicación con sus estudiantes. La Figura 9 describe la distribución porcentual de estas, siendo el correo electrónico, WhatsApp y Telegram los más usados.

\section{5 | Plataforma Tecnológica}

Una plataforma tecnológica ${ }^{4}$ es un sistema basado en un software bajo el que se exponen a través de Internet, contenidos virtuales a los usuarios(S. J. Rámos 2009) o LMS 5 La LMS de la UNAH opera bajo Moodle y permite acceso a comunicación síncrona a través de Zoom y de Team. El despliegue gráfico de la Figura 10, señala que más del $97 \%$ de los docentes usan la LMS de la UNAH para incluir contenido e indicar actividades asincrónas relacionadas con su clases.

\footnotetext{
${ }^{4}$ Learning Management System

${ }^{5}$ Learning Management System en inglés
} 


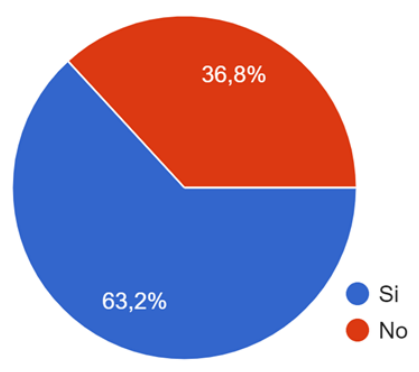

Figura 7: Uso de comunicación sincrónica.

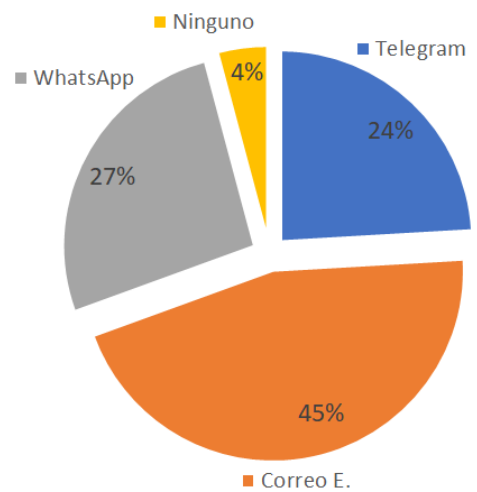

Figura 9: Herramientas complementarias de comunicación.

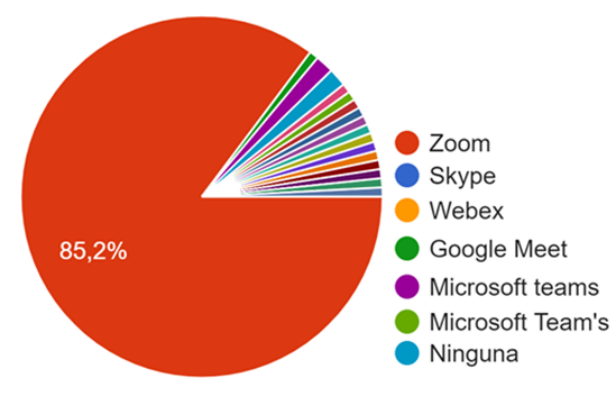

Figura 8: Uso de comunicación sincrónica.

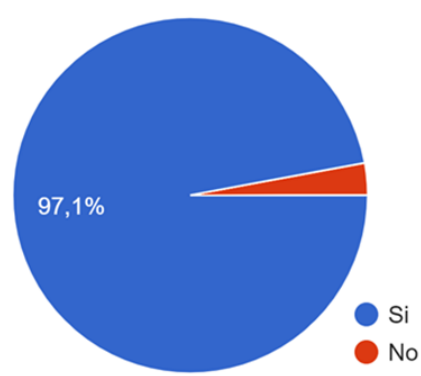

Figura 10: Uso de la LMS de la UNAH.

Las opciones de instrucción disponibles en la LMS-UNAH que más utilizan los docentes de esta área son: tareas, videos y pruebas en línea, seguidas de controles de estudio y material de lectura. Esto se indica en el histograma de la Figura 11

En cuanto a la efectividad de aprendizaje de estas herramientas, la gráfica de la Figura 12 indica la validación obtenida para cada una, evaluándose a los vídeos como los más efectivos, seguidos de tareas, lecturas, controles de lectura, controles de visualización de vídeos y las simulaciones 6 , según la opinión de los estudiantes de la muestra, los foros son los menos útiles en términos de aprendizaje.

Sobre la preferencia de modalidad y tecnología para la instrucción, la muestra consultada prefiere en un $86.44 \%$, que el docente use tanto tecnología de videoconferencia como la LMS-UNAH. Esto queda evidenciado en el despliegue gráfico de la Figura 13.

\footnotetext{
${ }^{6}$ Se recurrió a las simulaciones en computadora, para sustituir las experiencias de laboratorio.
} 


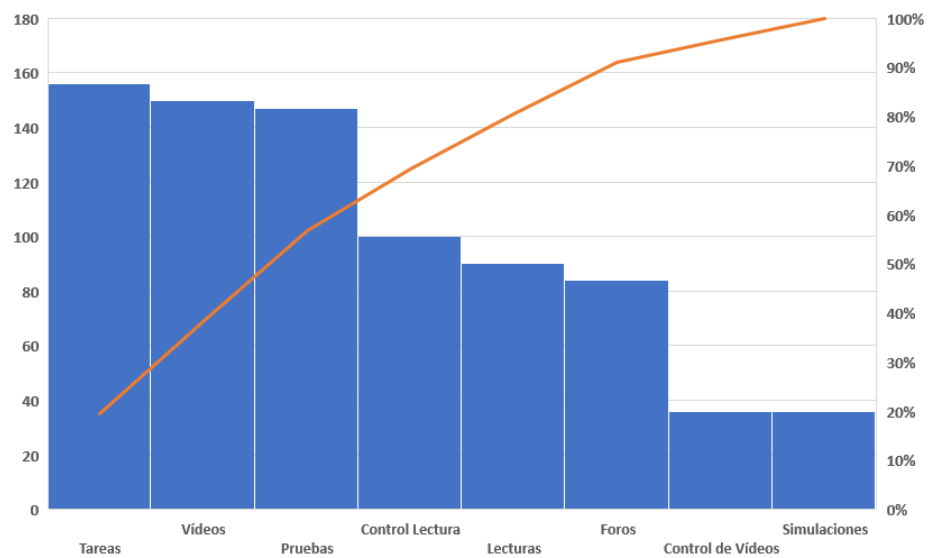

Figura 11: Opciones de Moodle utilizadas en la instrucción virtual.

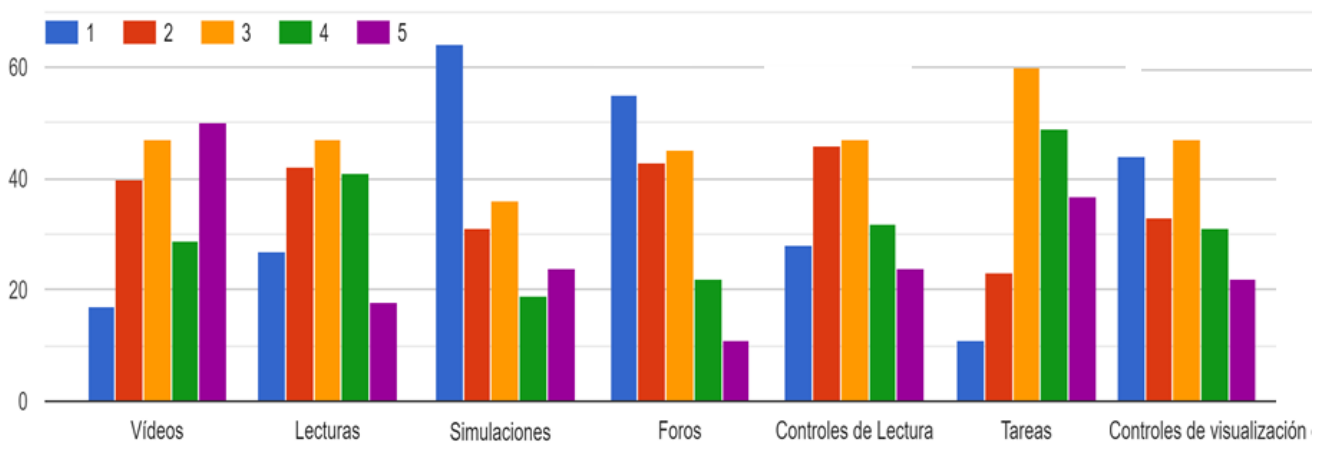

Figura 12: Potencial de aprendizaje de herramientas de instrucción.

\section{I Posibles Herramientas de Instrucción}

Se presentaron a la muestra cuatro (4) herramientas de instrucción, para evaluar su posible uso en los siguientes períodos académicos. Estas fueron:

- Pizarra virtual: software que permite utilizar la pantalla de la computadora como una pizarra y desplegar gráficos, ecuaciones y otros contenidos multimedia.

- Vídeo con control de atención: vídeo sobre un tópico, que plantea preguntas sobre el contenido expuesto, para evaluar el nivel de atención.

- Visualización de proceso escrito: una cámara (usualmente de un celular), permite a los estudiantes visualizar un procedimiento de solución resuelto por el docente en papel.

- Simulación explicada: uso de simulaciones en computadora sobre algún fenómeno físico, utilizado como complemento para detallar el desarrollo de un tema. 


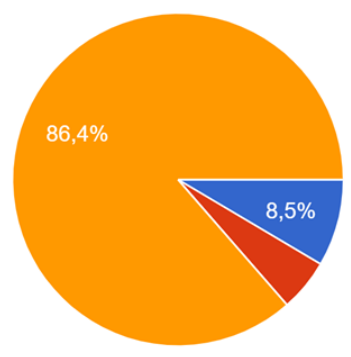

Que el docente SOLO haga uso de la Plataforma Virtual como herramienta para realizar su instrucción

Que el docente SOLO haga uso de tecnologia de video-conferencia e imparta su instrucción diariamente en el horario original de la clase

Que el docente use AMBAS opciones como herramientas de instrucción

Figura 13: Preferencias sobre la modalidad de instrucción.

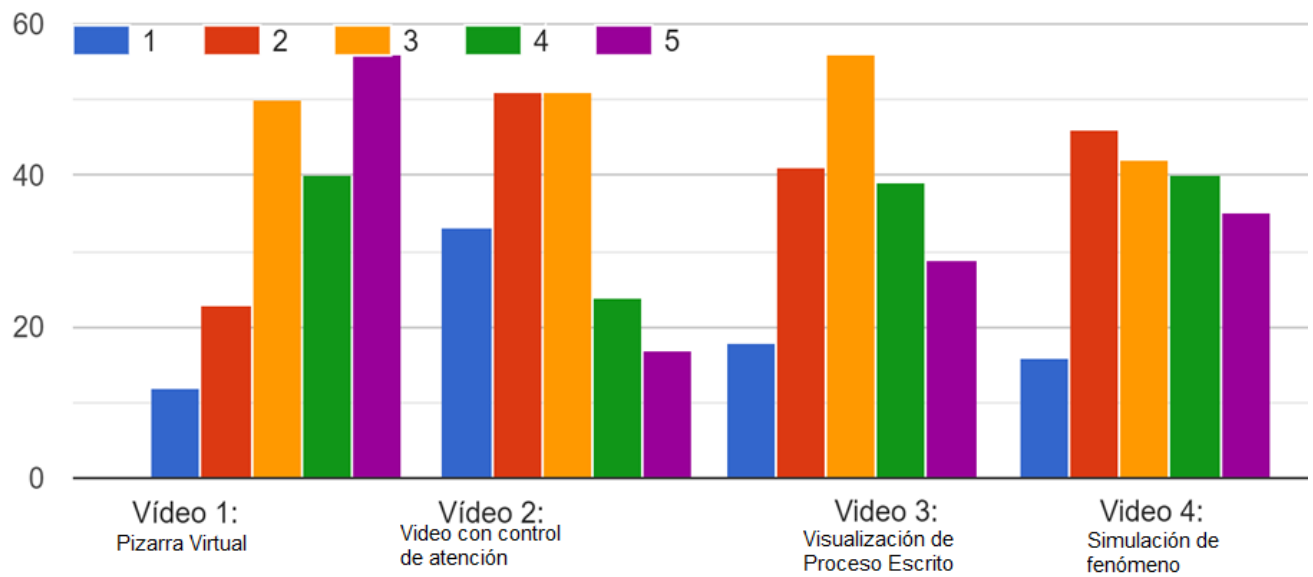

Figura 14: Futuras herramientas de instrucción.

La Figura 14 muestra el resultado de la validación de estas opciones por parte de los estudiantes consultados. Claramente ellos consideran con el mayor grado de efectividad para el aprendizaje a la pizarra virtual, seguida de la simulación explicada, y la visualización de proceso escrito la tercera; el video con control de atención incrustado resultó ser el de menor nivel de aprendizaje.

\section{7| Evaluación de LMS-UNAH}

Al solicitarles evaluar el desempeño de la LMS de la UNAH la respuesta fue la que se detalla en la Figura 15. El análisis de las respuestas de justificación con un algoritmo de minería de datos (librería Machine Learning NLTK de Python3)(Bengfort, 2016, Brownlee, 2017, Gonzáles, 2020), mostró que las palabras que más se repiten son las incluidas, junto con su frecuencia, en la Tabla 1

\section{I CONCLUSIONES}

- Casi el $71 \%$ de la muestra tiene acceso a computadora y casi un $29 \%$ se ve obligado a usar su celular para acceder a realizar sus actividades de instrucción. 


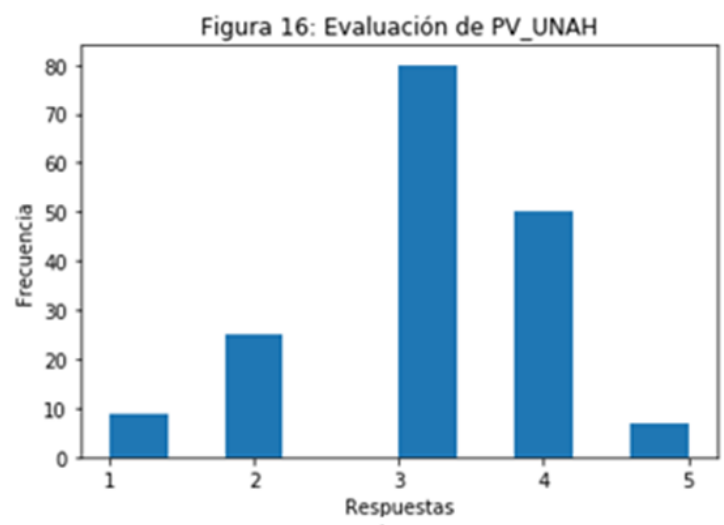

1: Pésima, 2: Mala, 3: Buena, 4: Muy Buena, 5: Excelente

Figura 15: Desempeño de LMS-UNAH.

\begin{tabular}{|c|c|c|c|}
\hline No.1 & Palabra & $\mathrm{f}$ & $\%$ \\
\hline 1 & $\mathrm{Cae}$ & 33 & \\
\hline 2 & Problemas & 21 & $76.14 \%$ \\
\hline 3 & Falla & 8 & \\
\hline 4 & Saca & 5 & \\
\hline 4 & Buena & 12 & $23.86 \%$ \\
\hline 5 & Bien & 9 & \\
\hline \multicolumn{2}{|c|}{ TOTALES } & 88 & $100.00 \%$ \\
\hline
\end{tabular}

- El $65 \%$ de los estudiantes tienen acceso a Internet sin problemas, mientras un porcentaje de casi el $32 \%$ tiene que hacer uso del celular.

- El 34.5\% de los estudiantes de la muestra gastan un monto de entre L20.00 a L200.00 semanales. Un $24.56 \%$ gasta entre L50.00 a L200.00 semanales.

- Los estudiantes que usan su celular para conectarse a internet, gastan entre L240.00 a L2400.00 por período.

- El $84.2 \%$ de la muestra, dice tener las competencias suficientes para afrontar esta modalidad de instrucción.

- El $63.16 \%$ de los docentes dan sus clases con algún servicio de vídeo conferencia y además recurren mayormente a WhatsApp, correo electrónico y Telegram para comunicarse con los estudiantes.

- Más del 97\% de los docentes usan la LMS de la UNAH para asignar actividades de instrucción. 
- Las herramientas de la Plataforma Virtual que los docentes usan, son en orden de frecuencia: Tareas, Vídeos y Pruebas, seguidas de Control de Lectura, Lecturas y Foros; la menos utilizada son las Simulaciones.

- En cuanto a la efectividad didáctica de estas herramientas, la muestra considera como más efectiva a los vídeos, seguidos de tareas, lecturas, controles de lectura, ubicándose al final los controles de visualización de vídeos y las simulaciones; opinan que los foros son los menos útiles en términos de aprendizaje.

- La herramienta de instrucción virtual que podría usarse y que la muestra consideró con mayor potencial de aprendizaje es la Pizarra Virtual, seguida de Simulaciones Explicadas, y la Visualización de Proceso Escrito.

- El desempeño de la LMS-UNAH fue malo en un 76.14\%. Explican que se satura en demasía, se cae con frecuencia y les genera problemas en exámenes y pruebas.

- Es importante mencionar que el área a la que pertenecían la muestra consultada, físico matemática, reúne a los estudiantes mejor preparados con los que trata el cuerpo docente de la Escuela de Física; son los que tienen las mejores competencias y experiencia en el uso de matemáticas y TICs.

- Los estudiantes perciben que las simulaciones de fenómenos físicos son buenas herramientas didácticas cuando son usadas por el docente mientras este explica los propiedades de un fenómeno. Cuando se les pide evaluar a éstas en el contexto en que han sido usadas durante la instrucción (laboratorios virtuales) su opinión las posiciona prácticamente en el último lugar de aprendizaje. Esto indica lo inadecuado, considerando la naturaleza experimental de la Física, de pretender sustituir las experiencias de laboratorio con simulaciones de los fenómenos estudiados.

\section{REFERENCIAS}

Acevedo, N. (2020). Tipos de gráficos con python. Obtenido de: https://nataliaacevedo.com/tipos -degraficos-en-python-con-matplotlib/

Bengfort, B. (2016). Applied text analysis with python. O'Reilly.

Brownlee, J. (2017). Deep learning for natural language processing. machine learning mastery.

Gallery, T. P. (2020). 20 basic histogram with seaborn. Obtenido de: https://python-graph-gallery.com/ 20-basichistogram-seaborn/

Gonzáles, C. G. (2020). Clasificador de texto mediante técnicas de aprendizaje automático.

S. J. Rámos, J. d. (2009). Una plataforma para gestión de clases virtuales. Revista Electrónica de Tecnología Educativa.

UNESCO. (2020). Evaluación y continuidad educativa en momentos difíciles. Autor.

y Roldán S. F., P. L. (2015). Metodología de la investigación social cuantitativa. UAB, Universitat Autónoma de Barcelona. 